\title{
Über die keimungsfördernde Wirkung verschiedener Acetaldehyd-Präparate
}

\author{
Von H. RUdolPH \\ Aus dem Botanischen Institut der Christian-Albrechts-Universität Kiel \\ (Z. Naturforschg. 16 b, 611-614 [1961]; eingegangen am 20. April 1961)
}

\begin{abstract}
Unvorbehandelte Sporen von Phycomyces blakesleeanus können durch verschiedene, handelsübliche Acetaldehyd-Präparate stark zur Keimung angeregt werden. Die Wirksamkeit eines Präparates ist allerdings sehr gering; die Ursache ist ungeklärt. Ebenfalls sind die Sporen durch Acetaldehyd aktivierbar, der unter bestimmten Versuchsbedingungen von wärmevorbehandelten Sporen in großer Menge gewonnen werden kann. Die Frage, auf Grund welcher Reaktionsfolgen die Sporen von Phycomyces blakesleeanus bei Acetaldehydzusatz zur Nährlösung keimen, muß vorerst unbeantwortet bleiben.
\end{abstract}

Unvorbehandelte Sporangiosporen von Phycomyces blakesleeanus keimen in definiertem Substrat im Durchschnitt zu 3,6\% (Sommer und Halbsguth $\left.1957^{1}\right)$. Unter anderem kann durch eine Wärmevorbehandlung der Keimprozentsatz auf mehr als 95\% erhöht werden (RoBbins und Mitarb. 1942 ${ }^{2}$, Sommer und Halbsguth $1957^{1}$, Halbsguth und RudolpH $1959^{3}$ ). Wärmevorbehandelte Sporen scheiden unter aeroben Bedingungen im Gegensatz zu unvorbehandelten Sporen kurzfristig Acetaldehyd in die Nährlösung aus ${ }^{4}$. Es war nun interessant zu prüfen, ob eine Verbindung, die nachweislich im Anschluß an die Wärmeaktivierung im Stoffwechselgeschehen auftritt, selbst unvorbehandelte Sporen zur Keimung anzuregen vermag. Außerdem haben die Untersuchungen von BrinckmanN $\left(1960^{5}\right)$ der Frage der Keimungsförderung der Sporen von Phy. comyces blakesleeanus durch Acetaldehyd einen weiteren Akzent gegeben. Auf Grund der gefundenen, relativ geringen Keimungsförderung (35\% Keimung bei Acetaldehydzusatz im Konzentrationsbereich 0,016-0,065-m.) mißt sie im Rahmen ihrer Untersuchungen zur Frage der Veränderung der Nährlösung durch Hitzesterilisation der keimungsfördernden Wirkung von Acetaldehyd nur eine untergeordnete Bedeutung zu. Auf die erheblich stärkere Wirksamkeit niedriger Acetaldehyd-Konzentrationen wurde von mir an anderer Stelle (RUDoLPH $1960^{4}$ ) bereits aufmerksam gemacht und eine eingehendere Darstellung in Aussicht gestellt. Darüber hinaus soll

1 L. Sommer u. W. Halbsguth, Forschungsberichte des Wirtschafts- und Verkehrsministeriums Nordrhein-Westfalen Nr. 411 (1957).

2 W. J. Robbins, V. W. Kavanagh u. F. Kavanagh, Bot. Gaz. 104, 224 [1942]. versucht werden, die Ursache, die zu einer so unterschiedlichen Beurteilung der keimungsfördernden Wirkung von Acetaldehyd führt, zu finden.

\section{Methode}

Als Maß für die Wirksamkeit von Acetaldehyd wurde der Keimprozentsatz (deutliche Vakuolenbildung) nach 12-stdg. Bebrütung in Standard-Nährlösung benutzt. Der Keimprozentsatz wurde mit einer Zählkammer nach $\mathrm{N}$ e u b a u e r bestimmt. Zur Grundlage der Methode vgl. Sommer und Halbsguth $\left(1957^{1}\right)$. Standard-Nährlösung: $15 \mathrm{~g}$ Glucose; $1 \mathrm{~g}$ Asparagin; $m / 100-\mathrm{KH}_{2} \mathrm{PO}_{4}$; $m / 1000-\mathrm{MgSO}_{4} \cdot 7 \mathrm{H}_{2} \mathrm{O} ; \quad m / 2000-\mathrm{Ca}\left(\mathrm{NO}_{3}\right)_{2} \cdot 4 \mathrm{H}_{2} \mathrm{O}$; $m / 10000-\mathrm{FeCl}_{3} \cdot 6 \mathrm{H}_{2} \mathrm{O} ; 1 / 100 \mathrm{\gamma} / \mathrm{ml}$ Vitamin $\mathrm{B}_{1}$; bidest. Wasser (Quarz) ad $1000 \mathrm{ml} ; p_{\mathrm{H}}=4,15 \pm 0,05$. Das Sporenmaterial (Dunkelkulturen) war $11 \pm 1$ Tage alt; Anzuchtstemperatur: $18 \pm 1{ }^{\circ} \mathrm{C}$; Anzuchtssubstrat: Vollkornbrot.

Der relativ niedrige Siedepunkt von Acetaldehyd muß bei der Versuchsanordnung durch Kühlung berücksichtigt werden! Von den Acetaldehyd-Präparaten wurden Stammlösungen mit eisgekühltem, quarz bidest. Wasser hergestellt. Diese Lösungen enthielten ca. $2000 \gamma$ Acetaldehyd $/ \mathrm{ml}$ und wurden bei $+1{ }^{\circ} \mathrm{C}$ aufbewahrt. Aus diesen Lösungen konnten die gewünschten Konzentrationsstufen hergestellt werden. Dabei war stets darauf geachtet worden, daß die Proben gut gekühlt waren. Die Bestimmung der in der Verdünnungsreihe in den einzelnen Stufen exakt vorhandenen Acetaldehydmenge erfolgte kolorimetrisch nach Sтотz $\left(1943^{6}\right)$. Durch Zugabe von $25 \mathrm{ml}$ gekühlter, doppelt konzentrierter, nicht sterilisierter Nährlösung zu einer Acetaldehydstufe von z. B. $200 \gamma / \mathrm{ml}$ ergab sich, daß $50 \mathrm{ml}$ StandardNährlösung $100 \gamma / \mathrm{ml}$ Acetaldehyd enthielt. Nach Ab.

3 W. Halbsguth u. H. Rudolph, Arch. Mikrobiol. 32, 296 [1959].

${ }^{4}$ H. Rudolph, Planta 55, 424 [1960].

5 M. Brinckmann, Arch. Mikrobiol. 37, 161 [1960].

6 E. Storz, J. biol. Chemistry 148, 585 [1943]. 
pipettieren von jeweils $5 \mathrm{ml}$ in 100-ml-Erlenm e ye r-Kölbchen mit Watteverschluß wurde mit unvorbehandelten, zweimal gewaschenen Sporen beimpft und bei $18^{\circ}+1{ }^{\circ} \mathrm{C}$ bebrütet. Die hier im folgenden genannten Acetaldehyd-Konzentrationen sind somit nur die jeweiligen Ausgangswerte; da sich in den Versuchskolben zum einen ein Gleichgewicht Flüssigkeit/Gasraum einstellt und zum anderen Acetaldehyd durch den Wattestopfen entweicht, sind die effektiv wirksamen Konzentrationen sicherlich noch geringer.

\section{Versuchsergebnisse}

1. Keimungsförderung durch industrielle Acetaldehyd-Präparate

Die Mittelwerte aus zahlreichen Keimversuchen sind in Abb. 1 dargestellt. Die Kurven a und b zeigen deutlich eine starke Keimungsförderung eines Präparates der Firma E. Merck und der Firma Riedel de Haën. Die Konzentration 0,00908-molar wirkt optimal fördernd auf die Vakuolenbildung. Höhere Konzentrationen machen sich ungünstig bemerkbar; dabei ist es nicht so, daß der Prozentsatz der Sporen ohne Vakuolen erheblich zunimmt, sondern die Vakuolen werden immer kleiner, so daß es schließlich unmöglich wird zu unterscheiden, ob eine Spore als gekeimt zu zählen ist oder nicht. Aus diesem Grunde ist in Abb. 1 der Konzentrationsbereich >0,017-molar nicht mehr berücksichtigt. Die Konzentration, die die Vakuolenbildung optimal fördert, wirkt nicht in gleichem Maße günstig auf die Keimschlauchbildung, denn nach 12 Stdn. Bebrütungszeit finden sich im Durchschnitt keine Sporen, die Keimschläuche ausgebildet haben. 0,0034-molare und geringer konzentrierte Lösungen fördern dagegen trotz der weniger günstigen Wirkung auf die Vakuolenbildung stark das Keimschlauchwachstum. Somit läßt sich auch der Acetaldehyd in die Gruppe jener Substanzen einordnen, die helfen, die Trennung von Vakuolen- und Keimschlauchbildung als zwei getrennte, entwicklungsphysiologische Prozesse zu stützen (Sommer und Halbsguth $1957^{1}$ ). Durch Vorbehandlung der Sporen mit Acetaldehyd kann im Gegensatz zum Acetat (Sommer und Halbsguth $1957^{1}$ ) allerdings nur eine geringe Förderung (maximal 10\%) erzielt werden.

Wie aber auch aus Abb. 1 c ersichtlich ist, reagieren die Sporen auf ein anderes Acetaldehyd-Präparat (E. Merck) nur sehr schwach. Die Ursache für dieses

7 J. E. Seegmiller, J. biol. Chemistry 201, 629 [1953].

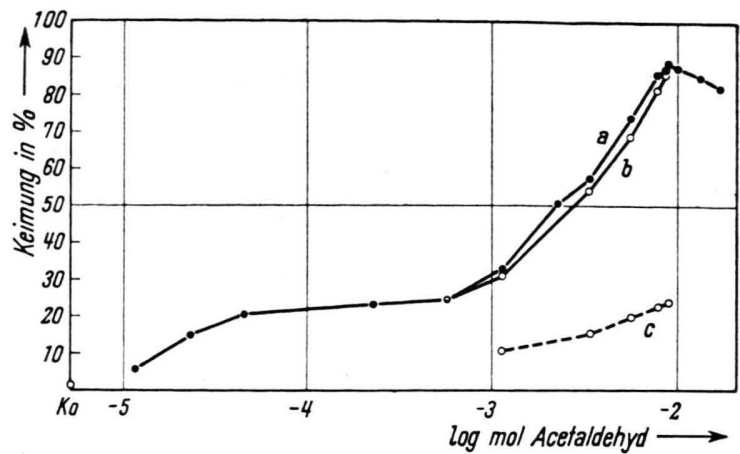

Abb. 1. Keimungsförderung unvorbehandelter Sporen durch Zusatz von industriellen Acetaldehyd-Präparaten zur Nährlösung. $\mathrm{Ko}=$ Kontrolle; $\mathrm{a}=$ Acetaldehyd (E. Merck) $; \mathrm{b}=$ Acetaldehyd (Riedel de Haën); $c=$ Acetaldehyd (E. Merck).

Verhalten konnte noch nicht ermittelt werden; möglicherweise enthielt dieses Präparat noch einen Hemmstoff.

Seegmiller $\left(1953^{7}\right)$ beobachtete bei seinen Untersuchungen zu einer an TPN gebundenen Acetaldehyd-Dehydrogenase aus Hefe, daß Verdünnungen von frisch dest. Acetaldehyd eine hemmende Wirkung ausüben, welche nach Aufbewahrung von 24. Stdn. bei $2{ }^{\circ} \mathrm{C}$ verschwinden! Eine entsprechende Beobachtung konnte bei den hier dargestellten Keimversuchen nicht gemacht werden.

Da es nun aber offensichtlich physiologisch verschieden wirksame Acetaldehyd-Präparate im Handel gibt, könnten die Ergebnisse von Sommer und Halbsguth (1957 ${ }^{1}$ ) und Brinckmann $\left(1960^{5}\right)$, die keine bzw. eine nur relativ geringe Förderung der Keimung unvorbehandelter Sporen durch Acetaldehyd zeigen, ihre Erklärung finden.

2. Die Aktivierung unvorbehandelter Sporendurch Acetaldehyd, dervon wärmevorbehandelten Sporen produziert wird

Wenn Sporen Acetaldehyd im Anschluß an eine Wärmevorbehandlung in die Nährlösung ausscheiden, dann müßte es möglich sein, unvorbehandelte Sporen mit diesem Acetaldehyd zu aktivieren. Zwar sind dazu die Acetaldehydmengen, die in StandardNährlösung nach einer Wärmevorbehandlung von 3 Min. bei 50 bzw. $54{ }^{\circ} \mathrm{C}$ ausgeschieden werden, zu gering; doch hat man die Möglichkeit, die Sporen nach einer Vorbehandlung bei $54{ }^{\circ} \mathrm{C}$ (3 Min.) und Überführung in eine Nährlösung, die $m / 10$ 000-2.4Dinitrophenol enthält, zu einer außerordentlich star- 
ken Acetaldehyd-Produktion anzuregen ${ }^{8}$ ! Dieser Acetaldehyd wird entsprechend der Versuchsanordnung Rudolph $\left(1960^{4}\right)$ gewonnen. Wird der Luftstrom, der mit dem ausgeschiedenen Acetaldehyd das Kulturrohr verläßt, nicht zum Abfangen des Acetaldehyds durch eine Bisulfitvorlage geleitet, sondern in ein mit Glasperlen gefülltes Absorptionsrohr, in dem sich Standard-Nährlösung befindet, die mit unvorbehandelten Sporen beimpft ist, so stellt sich entsprechend der Temperatur der beimpften Nährlösungsvorlage $\left(18^{\circ} \mathrm{C}\right)$ und der Strömungsgeschwindigkeit eine Acetaldehyd-Konzentration ein und die Sporen müßten zur Keimung angeregt werden. Dies ist der Fall! So keimten z. B. in einem Experiment $65 \%$ der eingeimpften Sporen, in der Kontrolle nur 1\%; das Keimschlauchwachstum war stark gefördert. 90\% Keimung, wie sie mit reinen Acetaldehyd-Präparaten erreicht werden kann, wurde allerdings nie beobachtet. Dies liegt sicherlich daran, daß die Konzentration bei der gewählten Temperatur von $18^{\circ} \mathrm{C}$ und der eingestellten geringen Strömungsgeschwindigkeit trotz eines reichlichen Acetaldehydangebotes nicht gesteigert werden kann und ein großer Teil des Aldehyds wieder aus der Nährlösung mit dem Luftstrom entweicht. In mehreren, solcher hintereinander geschalteten Absorptionsröhren konnten in einem Experiment durch den jeweils aus der vorgeschalteten Röhre wieder entweichenden Acetaldehyd 55\% bzw. 49\% der Sporen aktiviert werden.

Die Keimungsförderung der genannten industriellen Präparate beruht somit sicher auf der Wirkung des Acetaldehyds und ist nicht die Folge einer Verunreinigung mit einer anderen, stark keimungsfördernden Substanz; dafür spricht auch die starke Wirksamkeit der Präparate in so geringen Konzentrationen.

3. Deutung der Versuchsergebnisse

Bezüglich der Spezifität der Wirkung des außerordentlich reaktionsfreudigen Acetaldehyds sollen im folgenden 3 Möglichkeiten erwogen werden.

8 H. Rudolph, Planta, im Druck.

9 S. Black, Arch. Biochem. Biophysics 34, 86 [1951].

10 T. E. King u. V. H. Cheldelin, J. biol. Chemistry 220, 177 [1956].

11 St. W. Tanenbaum, Biochim. biophysica Acta [Amsterdam] 21, 335 [1956].

12 E. Racker, J. biol. Chemistry 177, 883 [1949].

13 F. H. Carpenter, Acta chem. scand. 5, 406 [1951].

14 A. H. Gordon, D. E. Green u. V. Subrahmanyan, Biochem. J. 34, 764 [1940].

\section{a) Acetaldehyd wird zu Äthanol reduziert}

Die Wirksamkeit des Acetaldehyds wäre dann ein „Äthanoleffekt“. Da aber vom Äthanol seit Sommer und Halbsguth $\left(1957^{1}\right.$ ) bekannt ist, daß es nicht keimungsfördernd auf unvorbehandelte Sporen von Phycomyces blakesleeanus wirkt, kann diese Möglichkeit ausgeschlossen werden.

\section{b) Acetaldehyd wird zu Essigsäure oxydiert}

Damit wäre die Acetaldehydwirkung letztlich eine Acetatwirkung. CoA-unabhängige Aldehyd-Dehydrogenasen sind mehrfach beschrieben worden. In Hefe: Black $1951^{9}$, Seegmiller ( $1953^{7}$, bei Acetobacter suboxydans bzw. peroxydans KING und Cheldelin $1956^{10}$, Tanenbaum $1956^{11}$ ); auch in tierischen Geweben fanden sich verschiedene Aldehyd-Dehydrogenasen z. B. RACKER (1949 12), CARpenter ( $\left.1951^{13}\right)$, Gordon, Green und SubrahmaNYAN $\left(1940^{14}\right)$.

Damit wären zusammen mit der Möglichkeit der Dismutation, bei der ein zweites Molekül Acetaldehyd als $\mathrm{H}_{2}$-Akzeptor dient, Wege aufgezeigt, nach denen es zu Oxydation von Acetaldehyd kommen könnte.

\section{c) Acetaldehyd geht direkt in den Stoffwechsel ein}

Äpfel und Orangen vermögen Acetaldehyd z. T. völlig zu $\mathrm{CO}_{2}$ zu oxydieren (Lit. vgl.: Thомаs $1956^{15}$ ).

Bäckerhefe ist in der Lage, Acetaldehyd zu einem großen Prozentsatz zu veratmen (GotrschalK $1941^{16}$ ). Bei Clostridium kluyveri wurde erstmals eine CoA-abhängige Aldehyd-Dehydrogenase gefunden (Stadtman und Barker 1949 ${ }^{17}$, Burton und Stadtman $1953^{18}$ ), unabhängig davon bei E. coli von Pinchot und Racker ( $\left.1951^{19}\right)$. Ob der reversiblen Dehydrierung, die Acetyl CoA liefert,

$\mathrm{CH}_{3} \mathrm{CHO}+\mathrm{CoASH}+\mathrm{PN}^{\oplus} \rightleftarrows$

$$
\mathrm{CH}_{3} \mathrm{CO}-\mathrm{SCoA}+\mathrm{PNH}+\mathrm{H}^{\oplus}
$$

eine allgemeine Verbreitung zukommt, ist noch unklar; es eröffnet sich jedoch nun möglicherweise ein

15 M. Thomas, S. L. Ranson u. J. A. Richardson, Plant Physiol., J. u. A. Churchill, London 1956.

16 A. Gotrschalk, Australian J. Biol. 19, 186 [1941].

17 E. R. Stadtman u. H. A. Barker, J. biol. Cchemistry 180, 1085 [1949].

18 R. M. Burton u. E. R. Stadtman, J. biol. Chemistry 202, 873 [1953].

19 G. B. Pinchot u. E. Racker, Phosphorus Metabolism 1, 366 [1951]. 
Weg zur direkten Veratmung von Acetaldehyd als Substrat. Die vorherige Umwandlung in Essigsäure vor der Einbeziehung in den Stoffwechsel würde dann einen „Umweg“ darstellen, denn von der Essigsäure darf angenommen werden, daß sie „nur in ihrer aktivierten Form chemische Reaktionsfähigkeit besitzt" (DECKER $1959{ }^{20}$ ).

Vergleicht man die Förderung durch Acetaldehyd mit der keimungsfördernden Wirkung von Acetat bzw. Ammoniumacetat wie sie von Sommer und HalbSGUth ( $1957^{1}$ ) angegeben wird, so stimmt die Konzentration, die eine 50-proz. Keimungsförderung bewirkt etwa überein. Bei diesem Vergleich darf aber nicht übersehen werden, daß der $p_{\mathrm{H}}$-Wert im Versuch mit Ammoniumacetat 7,1 bzw. 7,3 betrug, während bei den hier dargestellten Keimversuchen bei $p_{\mathrm{H}} 4,18$ experimentiert wurde. Überraschend stark ist die keimungsfördernde Wirksamkeit des Acetaldehyds in sehr geringen Konzentrationen. Acetaldehyd in der Konzentration 0,0000454-molar

20 K. Decker, Die aktivierte Essigsäure, F. Enke, Stuttgart 1959. bewirkt noch eine Keimung von 20 Prozent. Glyoxylsäure, die in ihrer keimungsfördernden Wirkung nach Brinckmann $\left(1960^{5}\right)$ die Essigsäure weit übertrifft, fördert in 0,0003-molarer Lösung noch 20\%; d. h. Acetaldehyd hat in einer noch um ca. eine Zehnerpotenz geringeren Konzentration die gleiche Wirkung. Bis heute ist keine Verbindung bekannt, die in so niedriger Konzentration noch in diesem Maße auf Phycomyces-Sporen keimungsfördernd wirkt.

Acetaldehyd besitzt somit eine die Keimung der Sporangiosporen von Phycomyces blakesleeanus stark fördernde Wirkung, denn zum einen können unvorbehandelte Sporen durch Acetaldehyd, den wärmevorbehandeltes Sporenmaterial unter bestimmten Versuchsbedingungen besonders intensiv produziert, aktiviert werden und zum anderen wird die Keimung unvorbehandelter Sporen durch industrielle Acetaldehyd-Präparate stark gefördert.

Der Deutschen Forschungsgemeinschaft sei für die Unterstützung gedankt, auch Frl. W. ReHDER für die Hilfe bei der Durchführung der Versuche.

\title{
Uber die optische Bestimmung der Sättigungskonzentration wasserlöslicher, kristallisierter Substanzen
}

\author{
Von Rudolf Mosebach \\ Aus dem Institut für Mineralogie und Petrologie der Justus Liebig-Universität Gießen \\ (Z. Naturforschg. 16 b, 614—618 [1961] ; eingegangen am 6. März 1961)
}

\begin{abstract}
Totalreflektometer und Refraktometer sind bei Einhaltung bestimmter Bedingungen zur Messung des Brechungsquotienten der kapillaren Flüssigkeitsschicht zwischen aufgelöstem Kristall und Glasvergleichskörper geeignet. Wählt man als Auflage einen wasserlöslichen Kristall und als optische Kontaktflüssigkeit Wasser, so vermittelt die beobachtete Lichtbrechungs-Variabilität der kapillaren Flüssigkeisschicht in Abhängigkeit von der Zeit die quantitative Kenntnis des Verlaufes der Lösungsreaktion bis zur Sättigungskonzentration. Die sinngemäße Abwandlung der Versuchsbedingungen führt zur Beantwortung einer Reihe von Fragen des Gleichgewichts zwischen Kristallen und kapillaren Lösungsschichten.
\end{abstract}

\section{Grundlage der Methode}

Die Bestimmung der Sättigungs-Konzentration kristallisierter Substanzen in Wasser läßt sich bei Anwendung einer geeigneten Versuchsanordnung auf optischem Wege leicht, schnell und genau durchführen.

Als Instrument diente bei den mitgeteilten Versuchen ein Totalreflektometer nach $\mathrm{Abbe-Czapski-}$ Pulf ri c h. Auch das A b b e - Refraktometer, das Präzisions - A b b e-Eintauch-Refraktometer, beide mit Kompensation für polychromatisches Glühlicht, und das Pulfrich-Refraktometer für Chemiker lassen sich verwenden. Wo die Glasvergleichskörper temperierbar sind, ergibt sich die Möglichkeit der Steigerung des Genauigkeitsgrades der Ergebnisse.

Das Prinzip der Messung ist sehr einfach. Man bringt auf die ebene Auflagefläche der Glashalbkugel des Totalreflektometers nach $\mathrm{Abbe-Czapski-Pulf-}$ $\mathrm{rich}$ einen von seinen gewachsenen ebenen Flächen begrenzten Kristall der Substanz, deren Sättigungs. Konzentration bestimmt werden soll. Wäre die Bestim. mung der Lichtbrechung dieser Kristallart das Ziel der Messung, dann müßte man die Kristallfläche mittels optischer Kontaktflüssigkeit mit der Halbkugelfläche derart verbinden, daß $n_{\mathrm{h}}>n_{\mathrm{f}}>n_{\mathrm{k}}$ ist. $\left(n_{\mathrm{h}}\right.$ bedeutet den Brechungsquotienten des Glases der Halbkugel 